\title{
Cómo conducir el interrogatorio en la presunta mujer estéril
}

\author{
Doctor Héctor Enrique Bernal B.
}

Profesor Agregado de Clínica Ginecológica de la Universidad Nacional. Ad_ junto del Departamento de Ginecología del Hospital de San José.

El primer paso en el estudio de una presunta mujer estéril io constituye el examen detenido y cuidadoso, tanto de su organismo en general, como de su aparato reproductor en particular. Este examen, que debe estar al alcance de cualquier ginecólogo, nos puede proporcionar de una vez por todas la causa de la presunta esterilidad, ya que generalmente se trata de lesiones groseras que afectan principalmente la esfera anatómica o de trastornos funcionales tan marcados que no escapan a la observación cuidadosa o al juicio clínico.

Sin embargo, lejos está de nosotros el tratar de aplicar un criterio tan simplista en tan complejo problema: como lo dice Stabile: "El hecho de hallar una alteración de esta indole durante el examen ginecológico no debe implicar nəcesariamente que sea ésta la única etiologia de una esterilidad; un cuello cónico, pongamos por caso, aunque intervenga como (llemento causal de esterilidad, debe ser tomado más a menudo como indice de una hipoplasia genital, y, por lo tanto, puede ser el efecto y no la causa de una afección mucho más compleja, donde la función ovárica y muy probablemente la de todo el sistema endocrino se halle en juego".

Como en cualquier otro ejercicio clínico, pero aquí, quizá más que en ninguna otra rama, un minucioso interrogatorio debe preceder al examen sin desdeñar ningún dato, por insigificante que parezca, que se relacione con el estado general, ya que existen diversas condiciones subclínicas que sin ningún efecto manifiesto sobre la historia menstrual o las funciones genitales pueden, sin cmbargo, afectar la fecundidad. No pecaríamos ce exagerados 
al recomendar en dicho interrogatorio una prudeneia inusitada: el penotrar en la vida afectiva de otra persona es junción delicada que asi como puede descubrirnos un amplio horizonte, puece, si no es ejercida con tacto, confundinos y aun hicernos perder la confianza del pacienú.

Edad: tiene importancia, ya que no ss infrusuente en la práctica, que la mujer consulte despues de los cuarenta años; y si bien es cierto que aun despues de esta edad pleden continuar produciéndose menstruaciones normales, no debemos olviciar que estamos en el umbral de la premenopausia, cuando el ovario tiende a reducir su actividad, una de cuyas caracteristicas es la producción cada vez más frecuente de ciclos menstruales anovulatorios.

En los antecedentes familiares interesa de marera espocial el grado de fecundidad en los ascendientes de los cónyuges, y particularmente la investigación de casos de esterilidad entre los hermanos casados de los mismos. Conviene tanbién investigar antecederites de endocrinopatias en la familia asi como de enfermedades hereditarias, y datos titiles desde ei punto de vista eugénico, tales como las infecciones- las intoxicaciones, los estados gererativos y las condiciones antihigiénicas de los progenitores (pauperismo, agotamiento físico, etc.), con la corsi. guiente productión de gametos de calidad inferior. cuva conjunción dará organismos constitucionalmente débiles, hasta é punto que se ha llegado a decir que "el porvenir sexual de estas mujeyes queda casi siempre determinado desde el momento de la fecundación, aunque puede ser modificado favorable o desfavorablemente durante los primeros años de la vida" (Anumada).

En efecto, y entrando en el estudio de los antecedentes personales, las enfermedades infecciosas agudas de la infancia al obrar sobre el delicado plasma germinativo pueden alterarlo en forma definitiva, y lo mismo podemos decir de las malas condiciones de vida de la niña antes de la pubertad. Entre las infecciones crónicas, conocido es el poder deletéreo de la tuberculosis y de la sifilis.

Más tarde quizá la lesión no sea tan marcada, pero el trastorno funcional puede ser intenso, ya que cualquier modificación del medio interno es capaz de influir desfavorablemente sobre el funcionamiento del ovario, exquisitamente sensible a toda influencia nociva. 
Dejando a un lado las causas endocrino-sexuales, cuyo estudio será objeto de un caṕtulo aparte, mencionemos rápidamente alguna de las afecciones que, pasada la pubertad, se encuentran con mayor frecuencia en los antecedentes. Aqu nuevamente las cnfermedades infecelosas agudas y crónicas (erisipela, escariatina, paperas, fieble tifoidca, estados septicémicos, tuberculosis, sifilis, etc.)

Mención especial merecen, sobre todo en la eulología de la estexilidad secundaria, las antecedeqnes quirúrgeos y traumáticus. de manera especial ias operaciones abdominomélvicas y vaginales. ES necesario precisar hasta donde sea posible el órgano sobre el cual se intervino y la ciase de intervencion practicada. Desafortunadamente en nuestro medio son muy pocaz las pacientes que pueden dar cuenta de elio; cuando más, se limitan a decir que fueron operadas de una anexitis, de un fibroma o de un quisto del ovario, etc., dejando planteado el interrngante. Naturalmente que no son ellas las únicas responsables. si es que de responsabilidad se puede hablar: mayor culpa tien: quizá el cirujano, que se contenta con dar vagos informes, cuando debiera consignar por escrito los detalles salientes de la intervención y las complicaciones, si las hubo. $Y$ ya que hablamos de complicaciones queremos llamar la atención sobre la importancia de averiguar si hubo drenaje post-operatorio que de por si nos indica la muy probable complicación peritoneal en el proceso, con formación de adherencias, etc., y sus posibles consecuencias en el tema que nos ocupa.

Continuando el interrogatorio debemos averiguar el régimen de vida de la paciente: como lo dice Lane-Roberts: "La manera de vivir en las ciudades tiende a deprimir la fertilidad en la mujer y esta tendencia se acrecienta en determinadas condiciones entre las cuales sobresalen la dieta inapropiada y la latiga". Todo extremo es vicioso, y así tanto la vida sedentaria como la demasiado intensa, pueden tener sus consecuencias.

Los factores dietéticos se reflejan muy claramiente en ciertos tipos de obesidad que se observa en pacientes subfértiles y que se caracterizan por depósitos adiposos en la región interescapular, en la cintura y en la parte interna de los muslos. Aun que asociado con deficiencias glandulares, este cuaúro no constituye un verdadero trastorno endocrino, y no responde a la terapia tiroidea u ovárica, cediendo, en cambio, al ajuste de la dieta; por consiguiente, debemos tomar el mayor cuidado en reconocer sus signos, ya que con frecuencia el cambio de dieta es el 
principal, si no el único recurso en la cura de la infertilidad. Queremos recordar a este respecto cuán frecuentes son entre nosotros los regímenes de base primordial de hidrocarbonados.

Mucho se ha hablado en los últimos años de la parte que corresponde a las vitaminas en el mantenimiento de la reproducción. La deficiencia de vitamina A puede inhibir el funcionamiento de la pituitaria y conducir a la interrupción de la secreción estrogénica. La vitamina $\mathrm{E}$ también es necesaria al normal funcionamiento del lóbulo anterior de la hipófisis, pars su influencia directa en la evolución de la gestación es aún de mayor significado. Se ha comprobado, en efecto, en los animajes, que una deficiencia severa de vitamina $\mathrm{E}$ induce a la muerte y reabsorción del feto por degeneración de la placenta; en la éspecie humana se cree que dicha deficiencia conduce al aborto. No hay razón para presumir que la deficiencia de vitamina $E$ sea responsable de la hipoplasia ovárica o de muchas otras condiciones para las cuales se la prescribe; pero existe alguna evidencia para creer que su administración a grandes dosis es capaz de contrarrestar el aborto habitual. Por consiguiente, el vaicr de la tera péutica con vitamina $\mathrm{E}$ es más de prevención que de curación $\mathrm{y}$ resulta difícil querer esperar de ella el aumento de las probabilidades de concepción; la razón quizá está en que a diferencia con los animales de experimentación, ia especie humana excepcionaimente sufre de esta carencia vitamínica; por otra parte, los requerimientos individuales varían grandemente y la dieta que sería adecuada para unos necesitaría ser adicionada en los otros.

En relación con las otras avitaminosis, nada concreto se sabe, aunque es probable que por su efecto sobre $e^{i}$ estado general de la mujer, al igual de otras enfermedades generales, disminuyan la capacidad para la concepción y sean asi causas indirectas de esterilidad. Agreguemos también cómo algunos autores han sugerido que los alimentos que crecen en terrenos fertilizados, principalmente con abonos químicos, sean deficientes en factores necesarios para la fertilidad.

El tratamiento dietético es necesario tanto en los casos de obesidad como de mala nutrición: las deficiencias, especialmen. te si se prolongan durante largo tiempo, tienden a producir sig. nos externos o trastornos de la función sexual, por ojemplo, amenorrea; pero aun cuando estos signos no aparecieran, la capacidad reproductora puede reducirse y la dieta debe ser equilibrada sin demora. Las medidas dietéticas especificas, tales como au- 
mento o reducción de un determinado alimento, constituyen un tema aparte, fuera de los límites de este resumen.

Es frecuente el caso de que las mujeres afectadas de esterilidad se entreguen al consumo de algunas drogas, en ocasiones por prescripción médica, pero que tomadas de manera continua o en exceso, pueden deprimir aún más su fertilidad. Tal es el caso del tiroides usado a menudo por pacientes obesas o ligeramente sobrepasadas de peso; sin embargo, un estudio de las curvas de temperatura y de las historias clínicas, demuestra que el uso excesivo o demasiado continuado de los preparados tiroideos puede inhibir la ovulación o deprimir la luteinización. De manera semejante muchas mujeres que han recibido prescripciones para estilbestrol o para otros estrógenos sintéticos continúan tomándolos durante largo tiempo, sin control del médico, en la creencia de que tratándose de la hormona iemenina no pueden esperar sino buenos resultados; ignoran, sin embargo, que el estilbestrol y otros estrógenos tienden a posponer la ovulación y que tomados en exceso pueden causar otros efectos indesables.

En nuestro país que como es sabido, es un "pais de drogas", en donde todo el mundo se medicina por su cuenta, y en donde inclusive en algunas regiones se destina una parte del salario para "la droga", es probable que en algunos casos se provoquen estados tóxicos transitorios que podrían perjudicar la fertilidad: entre ellos podríamos mencionar los abusos con las sulfas, la quinina, los compuestos arsenicales, etc.; especialmente en nuestros climas tropicales y en determinadas clases sociales. Sin embargo, lo repetimos, se trata en la mayoria de estos casos de esta cos transitorios; más importantes son, desde el punto de vista práctico, las severas intoxicaciones, capaces de producir infertilidad, a que por razón de su oficio pueden estar sometidas algu nas pacientess de manera continua: tal es el caso, por ejemplo de quienes trabajan con compuestos de plomo, o quienes con frecuencia reciben radiaciones. Este factor tóxico, importante entre nosotros casi exclusivamente en el hombre, cuiora, sin embargo, cada día mayor fuerza en la mujer, a medida que se produce su incorporación en la industria, y es posible que aún no sea mos capaces de reconocer el efecto que sobre la fertilidad tengan los numerosos venenos industriales, a los cuales se expone la mujer, aunque sus efectos sobre la hematopoyesis y otras funciones se hayan estudiado extensamente; algunos autores han demostrado que muchas de las ocupaciones industriales de la mujer aumentan la rata de morbilidad en su aparato reproductor. 
Al hablar de factores tóxicos es imprescindible mencionar el alcohol y el tabaco; no creemos, en realidad, que tlos constituyan un serio peligro para la fertilidad, salvo cuarıdo se consumen en cantidades enormes; su importancia, ya lo vimos al hablar del alcoholismo, estriba más en el profundo daño que puede causar al producto de la concepción que al proceso de la fertilidad en sí mismo.

Mencionábamos antes cómo las malas condiciones higiénicas durante la primera infancia podían afectar de una manera definitiva el plasma germinativo y determinar de una vez a la futura mujer estéril. Durante el período de madurez sexual la influencia nociva es mucho menor; pero, sin embargi, lo suficiente para aiterar la función y ocasionar esterilidad transitoria. Así, pues, no descuidemos en el interrogatorio detalles que pudieran parecer nimios en relación con las condiciones de habitación y de vida de la paciente: como lo dice Lane-Roberts: "No se deben sospechar únicamente agentes tóxicos, sino influencias tales como una postura defectuosa o una mala ventilación'.

Prácticamente desde el comienzo del interrogatorio se habrá ya definido si se trata de esterilidad primitiva o secundaria: es decir, si la falta de concepción ha sido absoluta o si, por el contrario, en época anterior hubo algún embarazo; ei este último caso debemos averiguar sobre el número de ellos y su evolución, insistiendo de manera especial en las posibles complicaciones del parto y el puerperio. Con frecuencia los embarazos terminados por abortos (sobre todo provocados), los partos o puerperios infecciosos dan origen a procesos ascendentes (endometritis, salpingitis, pelviperitonitis, etc.), que constituyen datos importantes para el diagnóstico de la esterilidad. En caso de que el embarazo hubiese llegado a término, con un parto normal, todavía debemos continuar nuestra investigación en el producto de la concepción: antecedentes tan importantes como la eritroblastosis fetal son en la práctica definitivos.

A propósito de los abortos es interesante averiguar si hubo logrado o no, aunque extraordinariamente raro, no debemos olvidar que los raspados realizados muy a fondo, en los que se extrae no sólo la funcional, sino también la capa basal del endometrio, pueden llevar a la enferma a la amenorrea definitiva.

Esto nos lleva a otra fase no menos importante del problema. Si con alguna frecuencia nos llegan enfermas que por primera vez consultan sobre su esterilidad, quizá no es menor el número de las que han pasado previamente por varios consul- 
torios, no siempre de especialistas. Insistiamos antes sobre la importancia de los antecedentes quirúrgicos de la paciente: alguno de ellos ha podido ser un tratamiento, dirigido inclusive a la esterilidad, que si por un lado eliminaba una causa, creaba en cambio, otra. Tal es el caso de las cauterizaciones y conizaciones del cuello demasiado profundas, de algunas estomatoplastias, etc. Pero no sólo los tratamientos, sino también algunas pruebas diagnósticas pueden haber contribuido a crear obstáculos a la fecundación. Sin querer restar en lo más mínimo los méritos indiscutibles de la histerosalpingografía como medio de averiguar la permeabilidad tubárica, queremos llamar la atención sobre la necesidad de limitar esta prueba a los casos en que esté absolutamente indicada. Motivo de capítulo especial será el estudio y discusión de dichos casos, así como el de los medios de contraste que deban emplearse; bástenos por ahora recordar, en relación con nuestro tema, cómo en algunas pacientes parece haberse acentuado $\mathrm{y}$ en ocasiones provocado una obstrucción que antes no existia, merced a la irritación provocada por un cuerpo extraño que tarda meses y a veces años $\in n$ eliminarse. Muchos son los especialistas que, con ocasión de intervenciones practicadas sobre las trompas, han encontrado como obstáculo principal a su permeabilidad la presencia de un contenido espeso, verdadero barro aceitoso, resto de una antigua salpingografía; sin mencionar la dificultad que al proceso mismo de la fecundación significa tan marcado cambio de medio.

Hemos dejado para lo último los antecedentes ginecológicos propiamente dichos, con lo cual queremos resaltar su importancia; de manera especial la historia menstrual de la paciente constituye el índice más valioso de que podemos disponer para conocer el funcionamiento del sistema endocrino-sexual femenino. El interrogatorio debe abarcar desde la menarquia hasta la última menstruación, insistiendo sobre la regularidad e intervalos entre las mismas, duración, cantidad de sangre, dolor, fenómenos asociados, etc. En la imposibilidad de extendernos en tema tan interesante, queremos recordar a grandes rasgos cómo la iniciación tardía de las reglas, asi como las crono-aromalias (poli y oligomenorrea), las hemorragias funcionales, la:s amenorreas sugieren la existencia de una insuficiencia del ovario, que se manifiesta generalmente por la incapacidad de concebir. Cuando tal insuficiencia es primaria, tiene gran importancia, pues indica casi siempre un estado de inferioridad más o menos grave del plasma germinativo, que clínicamente se traduce for la menar- 
quia tardia y la hipoplasia sexual, a veces acompañada de gran hipertrofia de los ovarios (ovarios micropoliquísticos), y finalmente por el agotamiento precoz del ovario o menopausia precoz: la esterilidad o la infertilidad de estas insuficientes ováricas, con hipoplasia secundaria de todo el sistema, ocupa un lugar importante en clínica ginecológica. Y ya que hablamos de Infertilidad recordemos la importancia que se debe dar a las modificaciones del ciclo menstrual después del matrimonio; de manera especial los retardos consecutivos, por cortos que sean, deben hacernos pensar en la posibilidad de una diskiesis: en efecto, muchos matrimonios no son estériles en el sentido exacto de la palabra, sino que permanecen infértiles a causa de los abortos de repetición. El nombre de diskiesis se aplica a la tendencia al aborto precoz que puede ocurrir en embarazos sucesivos sin causa claramente apreciable; en muchos casos no se sospecha dicha tendencia porque el aborto es tan precoz que simula una menstruación retardada. Su causa puede residir, ya en trastornos endocrinos o en una enfermedad localizada en el tracto genital femenino; puede también deberse a imperfecto desarrollo del corion, a su vez derivado de algún defecto constitucional de los gametos. La única forma de hacer el diagnóstico es mediante la determinación cuantitativa de las gonadotrofinas urinarias inmediatamente se presenta el retardo menstrual.

Nos prolongaríamos demasiado si insistiéramos en otras condiciones asociadas al flujo menstrual, como la dismenorrea, las jaquecas, los edemas, el dolor en los senos, etc. con frecuencia resultado de alteraciones en el equilibrio hormonal; queremos, en cambio, resaltar la importancia de tratar de establecer con ıa mayor precisión las características del ciclo en los seis últimos meses, única base que nos permitirá continuar con exito nuestro estudio.

Casi tan importante como los trastornos del cicio menstrual es el antecedente de leucorrea, índice en muchas ocasiones de una insuficiencia ovárica y las más de las veces de la invasión del canal genital o de sus anexos por un agente patógenc, capaz en algunas circunstancias de crear un medio hostil al espermato zoide y en otras de impedir el proceso de fecundación. Debe ser investigado cuidadosamente $\mathrm{y}$ tratado como paso preliminar antes de practicar otras pruebas, de manera especial las de permeabilidad de las vías genitales superiores.

Al llegar a esta etapa del interrogatorio nos jueda por estudiar un tema, si no más, por lo menos tan importante como 
los anteriores: nos referimos a la historia sexual de la paciente, o mejor, de la pareja matrimonial. Punto éste, inoudablemente el más delicado de investigar; requiere como condicićn indispen. sable el habernos ganado previamente la confianza absoluta de la enferma; de ahí que en muchas ocasiones, tal vez en la mayoria, no sea prudente abordarlo en la primera consuita. sino en consultas posteriores, aprovechando el momento oportuno, para de ahí en adelante proseguir con la mayor sutileza, recordando en todo momento que una pregunta indiscreta podria herir en lo más intimo la susceptibilidad de la paciente. Así, pues, preguntaremos sobre la aparición de la líbido, la frectiencia $y$ forma en que se realizan las relaciones sexuales, su relación con el ciclo, los caracteres del orgasmo y el grado de saisfacción obtenida, la presencia de doior, etc. Es un hecho sabido que el embarazo puede en algunas ocasiones seguir a la siraple eyaculación del semen en la vulva, sin que sea necesario para que se produzca la concepción ni el orgasmo femenino, ni la penetración dei pene en la vagina; pero también es iguaimente cierto que la focundación encuentra sus mejores condiciones cuando depositada la esperma en la vecindad del orificio cervical, la mujer experimenta una satisfacción sexual complota. Según algunos autores, el orgasmo femenino, paralelo con ei del marido o inmediatamente anterior a él, despierta en el útero y la vagina contracciones especiales, como resultado de las cuales el semen depositado en el iago seminal pasa al canal del cuello. En realidad no se trata de un fenómeno general, y si es cierto que observaciones aisladas lo han confirmado. estudios más recientes demuestran que esta especie de succión es excepcional, y que ei ascenso de los espermatozoides no cepende de acción mecánica tan simple. Es probable, sin embargo, que si la esperma se deposita cerca al cérvix el tránsito de los espermatozoides hasta su meta final se acorta enormemente en su parte más peligrosa, vale decir, en su paso a través de la vagina, ya que se ha probado que la célula espermática encuentra las condiciones màs favorables para permanecer viva y activa por largo tiempo en la vecindad del canal cervical, mientras que muere rápidamente en el medio vaginal. La duración exacta de su vida en estas regiones ha sido estimada diferentemente por los distintos observadores, pero todos convienen en que la gran mayoría de los espermatozoides vienen a ser inertes después de dos horas de permanencia en la vagina, mientras que los que han llegado al canal cervical pueden mostrar movimientos activos hasta cinco cías después; 
por consiguiente, es muy probable que cualquier condición que favorezca su paso rápido de la vagina al canal cervical aumente las probabilidades de embarazo.

Es también cosa aceptada que la preparación adecuada de la mujer para el coito, independientemente de su accion favorable a la producción del orgasmo, contribuye a crear las mejores condiciones para el espermatozoide. Si recordamos que la secreción cervical normal no sólo constituye el medio óptinso para ellos, sino que es necesaria para obtener una buena inseminación y por ende ia fecundación, comprenderemos cómo una buena corte, antes de la cópula, al excitar la secreción cervical crea las condiciones más favorables para el ascenso de la célula espermática. Infortunadamente son muchos los maridos que pasan por alto estos detalles, sin darse cuenta de que el amor físico es un arte; $y$, descansando en la creencia de que todo es producto de la saluci y del instinto, atribuyen la falta de placer sexual on su mujer a frigidez, cuando ia falta está en ellos. Cualquier médico que haya tenido que tratar con problemas sexuales matrimoniales observa con frecuencia cuán pocas son las parejas perfectamente compatible's y si interroga a las esposas son muchas las que confiesan no haber experimentado jamás el orgasmo.

Mención especialísima merecen las prácticas anticoncepcionales y los vicios sexualies: por un mecanismo infeccioso directo o indirectamente por modificación del medio normal de la vagina, pueden provocar estados de esterilidad transitorios. Pero quizá su acción más perniciosa reside en las profundas alteraciones de orden neurovascular que, debido a la falta de satisfacción sexual completa o a simple acción mecánica, sueler: determinar en la mujer. Las neurosis genitales son más frecuentes de lo que se sospecha: por el estado de congestión sanguínca y linfática pelviana que originan, constituyen la base etiopatogénica de estados patológicos no siempre bien interpretados, entre los cuales la dispareunia y concurrentemente la esterilidad son unos de los más conocidos.

Los límites de este trabajo nos impiden extendernos sobre tema tan apasionante, que, por lo demás, será ampiiado en el capítulo correspondiente al factor psicosomático, pero no podriamos dejar de mencionar una de las perturbaciones más conocidas de la cópula: nos referimos al vaginismo, verdadero reflejo muscular, caracterizado por el espasmo doloroso de los músculos transverso profundo del periné, bulbo-cavernoso y elevador del ano, desencadenado ante la menor tentativa de aproximación 
sexual y acompañado a veces de zonas de hiperalgesia de la fosa navicular y de la zona himeneovaginal vecina, así como también de la contractura refleja e invencible de los músciios aductores del muslo. En la mayoria de estas enfermas, con frecuencia neuróticas, se trata solamente de un reflejo defensivo francamente patológico, difícil de explicar, y solamente en un número reducido de casos, de un fenómeno involuntario provocacio por el choque del pene con una lesión erosiva o dolorosa del veetibulo o del himen.

\section{BIBLIOGRAFIA}

Ahumada Juan C.--“"Tratado Elemental de Ginecologia”. Tercera edición. Buenos Aires. 1952.

Amaya León Hernando.- "Tuberculosis en Ginecología". "Revista Colombiasa de Obstetricia y Ginecología". Vol. I. № 6. Octubre-noviembre 1950.

Bernal Héctor Enrique.-"Miomatosis y Embaraz". "Cirugía conservadora". Repertorio de Medicina y Cirugía. Bogotá. Vol. V. No 2. Enero-febre. ro 1950 .

Buxton C. L. and Matthews C. S.-"Bacteriology of the Cervix in cases of Infertility". Fertility and Sterility. Vol. 2. No I. Jan. 1951.

Calatroni Carlos J. y Ruiz Vicente- "Terapéutica Ginecológica". 3? edición. "El Ateneo".. Buenos Aires. 1944.

Conill M. Victor.-."Tratado de Ginecologia". Barcolona. 1043.

Cotte Gaston.- "Troubles Fonetiona?es de LAppaureil Genital de la Femme". Masson et Cie. París. 1931.

Hamblen. E. C.- "Endocrinology of Woman". Charles C. Thomas. Springfield III. 1950.

Curtis Arthur Hale.-."Ginecología". Barcelona. 1941.

Jenser K. M. and Mc. Donald J. R. “Am. J. Obst. - Gynec." Vol. 41. pág. 268. 1941.

Lane-Roberts Cedric.- "Sterility and Impaired Fertility". Paul B. Hoeber Inc. Second edition. Iondon. 1948. 1934.

Meaker S. R.-"Human Sterity". Baltimore, Williamns ind Wilkins Co.

Notas Médicas (Schering Corporation).-Vol. II. No \&. Ecpt. 19!?.

Stabile Américo.- "La Esterilidad Matrimonia1". Buenos Aires. "El Ate. neo". 1947.

Taylor Howard. C. Jr.-_Vascular Congestion and Hyperemia". Am. J. of Obst_Gynec. Vo!. 57. Numbers 2 and 4. 1949.

Taylot Howard C. Jr. "Life Situations, Emotions and Gynecologic pain Associated With Congestion". 